\section{Induction of the Abscopal Effect with Immunotherapy and Palliative Radiation in Metastatic Head and Neck Squamous Cell Carcinoma: A Case Report and Review of the Literature}

\author{
Ashwin Shinde ${ }^{1}$, Jennifer Novak ${ }^{1}$, Morganna L. Freeman ${ }^{2}$, Scott Glaser ${ }^{1}$, Arya Amini ${ }^{1}$ \\ 1. Radiation Oncology, City of Hope National Medical Center, Duarte, USA 2. Medical Oncology, City of Hope National \\ Medical Center, Duarte, USA
}

Corresponding author: Ashwin Shinde, adshinde@coh.org
Received 12/17/2018

Review began 01/03/2019 Review ended 02/21/2019 Published 03/07/2019

\section{() Copyright 2019}

Shinde et al. This is an open access article distributed under the terms of the Creative Commons Attribution License CC-BY 3.0., which permits unrestricted use, distribution, and reproduction in any medium, provided the original author and source are credited.
Categories: Otolaryngology, Radiation Oncology, Oncology

Keywords: head and neck cancer, palliative, abscopal effect, quad shot, immunotherapy, ctla-4, pd-1 inhibitor, radiation

\section{Introduction}

Metastatic head and neck squamous cell carcinoma (mHNSCC) is a difficult disease to treat, with poor prognosis and median survival of between 6 and 12 months [1]. Standard therapy over the past decade has been a combination of cetuximab, cisplatin, and 5 -flurouracil, albeit with significant toxicity and response rates of approximately $33 \%$ [2]. More recently, there has been significant interest in the use of immunotherapy in the management of mHNSCC [3]. Treatment with immunotherapy introduces discussion about the potential for the abscopal effect when combined with radiation. The abscopal effect was first described in the 1950s, and is seen with radiation, where a reduction in disease occurs outside the radiated site, through primarily immune mediation [4]. The most well-known case report of the abscopal effect was in a melanoma patient receiving ipilimumab who stopped responding after years of stable disease and developed progressive disease. The patient received palliative radiation, and subsequently showed partial responses in both the irradiated and unirradiated lesions [5]. Since then, there has been significant interest in combining immunotherapy with radiation to induce the abscopal effect, initially in mice, then across multiple disease sites [6-7]. We present a case of the abscopal effect observed in a patient with mHNSCC.

\section{Case Presentation}

A 75-year-old male former smoker with 45-pack year history of tobacco abuse initially presented with swelling of the left neck, followed by progressive hoarseness and dysphagia over a course of one to two months. A biopsy of the left neck node demonstrated squamous cell carcinoma (SCC), p16 positive. The patient also had a CT scan and follow-up positron emission tomography-CT (PET-CT) showing a $4.5 \mathrm{~cm} \times 7$ $\mathrm{cm}$ mass involving the left hypopharynx and oropharynx, crossing midline and causing narrowing of the hypopharyngeal and supraglottic airway. There was also bulky left level II neck lymphadenopathy measuring up to $7 \mathrm{~cm}$ in diameter. The patient was also found to have three fluorodeoxyglucose (FDG) avid lung nodules as well, one of which was subsequently biopsied, confirming metastatic p16 positive SCC. The patient required tracheostomy and gastrostomy placement for threatened airway obstruction and dysphagia, respectively. He was initially enrolled on a clinical trial comparing first-line EXTREME chemotherapy versus ipilimumab and nivolumab, and was randomized to combination ipilimumab and nivolumab delivered concurrently every three weeks for four cycles. Follow-up imaging at eight weeks showed progression of disease, with growth of the primary and neck masses ulcerating through his skin (Figure $1 \mathrm{~A}$ ), as well as disease progression in the chest, with growth of previous nodules and development of additional pulmonary nodules, the largest measuring $2.4 \mathrm{~cm}$ (previously $1.6 \mathrm{~cm}$, Figure $1 B$ ).

The patient had worsening symptoms associated with mass effect in the neck and was re-evaluated by Radiation Oncology at the request of the treating oncologist. He was treated with 'QUAD SHOT' for palliation [8], delivering 3.7 Gy BID x 2 days (total dose 14.8 Gy) to gross disease, and 3.3 Gy BID x 2 days $(13.2 \mathrm{~Gy})$ to microscopic areas at high risk for disease, including one nodal echelon beyond gross disease on the left (levels IB-V), and contralateral levels II-IV. No radiation was delivered to his lung disease. The 


\section{Cureus}

patient continued through the third and fourth cycles of nivolumab and ipilimumab without breaks or delays. Radiation was delivered during the three-week time frame between immunotherapy cycles, and he tolerated treatment well with minimal pain and noticeable improvement in the left neck mass.

On follow-up CT scan at two weeks after completion of radiation, the primary and bulky left neck adenopathy had decreased in size by approximately $25 \%$ (Figure 1 C). The metastatic pulmonary nodules had also all decreased in size with the largest nodule decreasing from 2.4 to $1.3 \mathrm{~cm}$, approximately $50 \%$ (Figure 1D). His QUAD-SHOT regimen was repeated one month after initial treatment, as described above. Repeat imaging showed continued decrease in size of all neck and most lung disease, with one lung nodule minimally increasing in size. The patient received a third round of QUAD-SHOT as described above, with near complete resolution of his left neck mass. At 16 weeks from first round of radiation treatment, the patient had a continued response in his pulmonary metastases, followed by disease stability. At 20 weeks from start of radiation, one of his lung lesions had increased in size while the remaining lesions were either stable or decreased in size. At the time of this writing (10 months from initial diagnosis), he has completed a total of 14 cycles of ipilimumab and nivolumab on trial and is currently living with stable, asymptomatic disease.

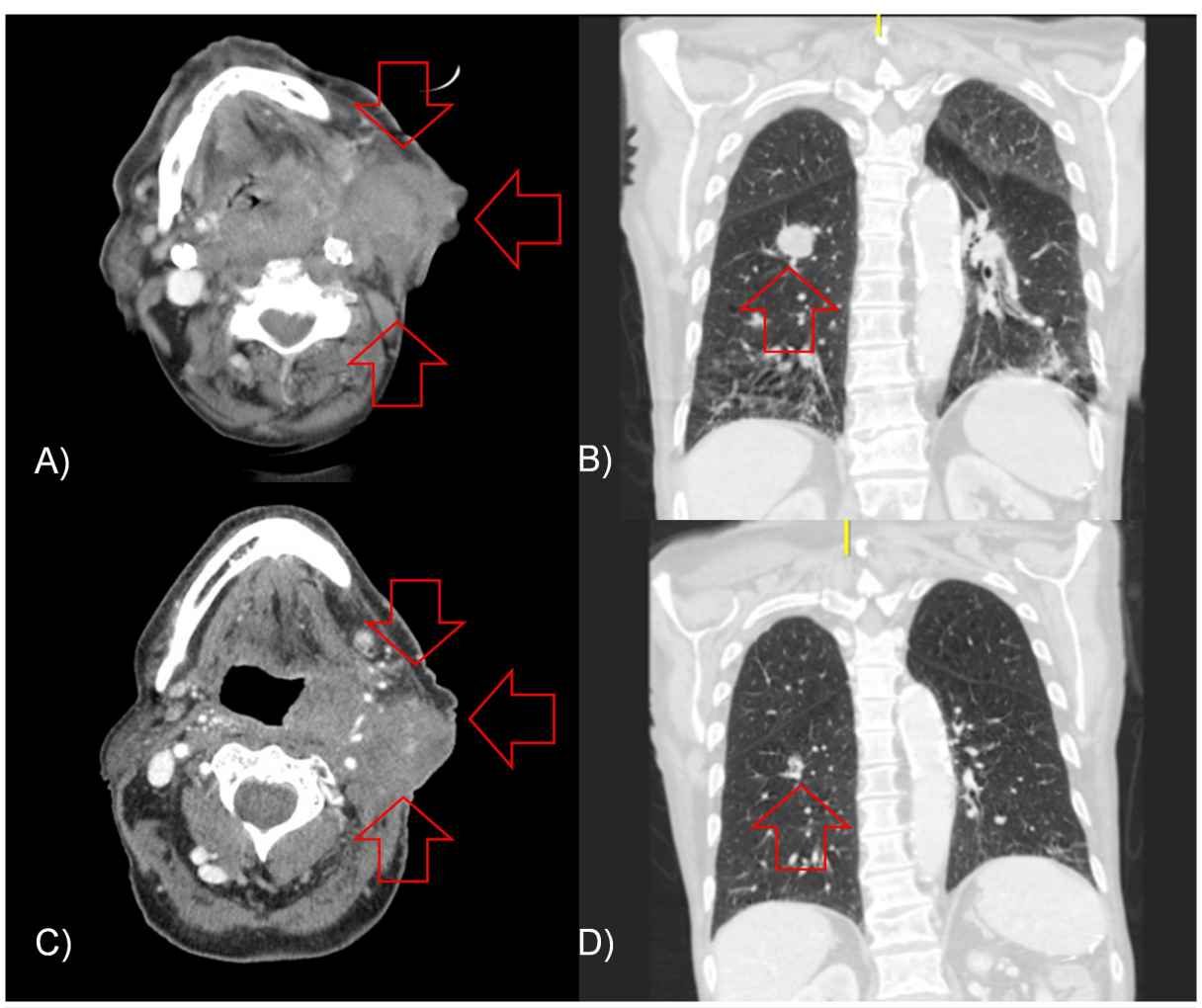

\section{FIGURE 1: Neck and chest imaging before and after QUAD-SHOT with} immunotherapy.

CT imaging of the patient demonstrating (red arrows) (A) axial images of his primary disease and left neck adenopathy, and (B) coronal images of his largest pulmonary nodule, along with treatment response two weeks after completing QUAD-SHOT radiation treatment in the (C) axial and (D) coronal planes.

\section{Discussion}

This case represents, to our knowledge, the first reported case of the abscopal effect in mHNSCC. Our patient did not have an initial response to combination ipilimumab and nivolumab immunotherapy after four cycles of therapy. He received palliative treatment using QUAD-SHOT, a high dose per fraction treatment that provides short treatment times, acceptable toxicity, and ability to repeat treatment, dependent on patient's prognosis and level of symptomatic relief [8]. After this course of palliative radiation, the patient showed partial response in both the irradiated neck and the unirradiated chest.

No previous cases of the abscopal effect in mHNSCC have been reported in the literature. Objective response rates (ORRs) with immunotherapy alone in mHNSCC are low. When used as second-line therapy with progression on platinum-based chemotherapy, single agent nivolumab has an ORR of 13.3\% [9], single agent pembrolizumab has an ORR of $18 \%$ [10], and single agent durvalumab has an ORR of 6.5\%-16.2\% [1112]. Combination therapies evaluated include durvalumab and tremelimumab (ORR 7.8\%) [13]. While the 
results of Checkmate-141 [9] showed improved survival compared to second-line single-agent chemotherapy in a phase III setting, additional studies are required to determine whether immunotherapy can lead to better survival than combination cisplatin, cetuximab, and 5-flurouracil as first line therapy for mHNSCC.

Results from currently ongoing trials may assist in future management decisions. KEYNOTE-040 will evaluate pembrolizumab in a phase III fashion against second-line single agent chemotherapy (Clinicaltrials.gov \#NCT02252042). Another trial is evaluating second-line cyclophosphamide, avelumab, and single fraction radiation of $8 \mathrm{~Gy}$ in mHNSCC [14]. A French group is evaluating combining durvalumab and tremelimumab with stereotactic body radiation (SBRT) in mHNSCC patients [15]. Additional studies evaluating the role of immunotherapy in mHNSCC include combining SBRT with nivolumab (Clinicaltrials.gov \#NCT03539198), comGiven poor ORRs of systemic immunotherapy alone in mHNSCC, lack of other acceptable treatment options, and poor prognosis of this patient population, we present this case report primarily for hypothesis generation; clinicians may consider combining immunotherapy with radiation in mHNSCC in an attempt to improve ORRs and potentially improve oncologic outcomes.bining nivolumab with experimental medication BMS-986205 and comparing it to cisplatin, cetuximab, and 5fluorouracil (Clinicaltrials.gov \#NCT03386838), combining pembrolizumab with clopidogrel (Clinicaltrials.gov \#NCT03245489), and combining pembrolizumab with afatinib (Clinicaltrials.gov \#NCT03695510).

Given poor ORRs of systemic immunotherapy alone in mHNSCC, lack of other acceptable treatment options, and poor prognosis of this patient population, we present this case report primarily for hypothesis generation; clinicians may consider combining immunotherapy with radiation in mHNSCC in an attempt to improve ORRs and potentially improve oncologic outcomes.

\section{Conclusions}

Our patient exhibited evidence of the abscopal effect when doublet immunotherapy did not induce a response, palliative radiation was added, and the combination led to partial response in both irradiated and unirradiated sites of disease. We feel that this case report is hypothesis generating for possibly combining immunotherapy and radiation to improve objective response rates and other oncologic outcomes in patient's mHNSCC.

\section{Additional Information}

\section{Disclosures}

Human subjects: Consent was obtained by all participants in this study. Conflicts of interest: In compliance with the ICMJE uniform disclosure form, all authors declare the following: Payment/services info: All authors have declared that no financial support was received from any organization for the submitted work. Financial relationships: All authors have declared that they have no financial relationships at present or within the previous three years with any organizations that might have an interest in the submitted work. Other relationships: All authors have declared that there are no other relationships or activities that could appear to have influenced the submitted work.

\section{References}

1. Samra B, Tam E, Baseri B, Shapira I: Checkpoint inhibitors in head and neck cancer: current knowledge and perspectives. J Investig Med. 2018, 66:1023-1030. 10.1136/jim-2018-000743

2. Vermorken JB, Mesia R, Rivera F, et al.: Platinum-based chemotherapy plus cetuximab in head and neck cancer. N Engl J Med. 2008, 359:1116-1127. 10.1056/NEJMoa0802656

3. Guidi A, Codecà C, Ferrari D: Chemotherapy and immunotherapy for recurrent and metastatic head and neck cancer: a systematic review. Med Oncol. 2018, 35:37. 10.1007/s12032-018-1096-5

4. Formenti SC, Demaria S: Systemic effects of local radiotherapy . Lancet Oncol. 2009, 10:718-726. 10.1016/S1470-2045(09)70082-8

5. Postow MA, Callahan MK, Barker CA, et al.: Immunologic correlates of the abscopal effect in a patient with melanoma. N Engl J Med. 2012, 366:925-931. 10.1056/NEJMoa1112824

6. Deng L, Liang H, Burnette B, Beckett M, Darga T, Weichselbaum RR, Fu YX: Irradiation and anti-PD-L1 treatment synergistically promote antitumor immunity in mice. J Clin Invest. 2014, 124:687-695. 10.1172/JCI67313

7. Abuodeh Y, Venkat P, Kim S: Systematic review of case reports on the abscopal effect . Curr Probl Cancer. 2016, 40:25-37. 10.1016/j.currproblcancer.2015.10.001

8. Corry J, Peters LJ, Costa ID, Milner AD, Fawns H, Rischin D, Porceddu S: The 'QUAD SHOT'-a phase II study of palliative radiotherapy for incurable head and neck cancer. Radiother Oncol. 2005, 77:137-142. 10.1016/j.radonc.2005.10.008

9. Ferris RL, Blumenschein G, Fayette J, et al.: Nivolumab for recurrent squamous-cell carcinoma of the head and neck. N Engl J Med. 2016, 375:1856-1867. 10.1056/NEJMoa1602252

10. Seiwert TY, Burtness B, Mehra R, et al.: Safety and clinical activity of pembrolizumab for treatment of recurrent or metastatic squamous cell carcinoma of the head and neck (KEYNOTE-012): an open-label, multicentre, phase 1b trial. Lancet Oncol. 2016, 17:956-965. 10.1016/S1470-2045(16)30066-3

11. Zandberg DP, Algazi AP, Jimeno A, et al.: Durvalumab for recurrent or metastatic head and neck squamous cell carcinoma: results from a single-arm, phase II study in patients with $\geqslant 25 \%$ tumour cell PD-L1 


\section{Cureus}

expression who have progressed on platinum-based chemotherapy. Eur J Cancer. 2019, 107:142-152.

10.1016/j.ejca.2018.11.015

12. Segal NH, Ou S-HI, Balmanoukian A, et al.: Safety and efficacy of durvalumab in patients with head and neck squamous cell carcinoma: results from a phase I/II expansion cohort. Eur J Cancer. 2019, 109:154-161. 10.1016/j.ejca.2018.12.029

13. Siu LL, Even C, Mesía R, et al.: Safety and efficacy of durvalumab with or without tremelimumab in patients with PD-L1-low/negative recurrent or metastatic HNSCC: the phase 2 CONDOR randomized clinical trial. JAMA Oncol. 2019, 5:195-203. 10.1001/jamaoncol.2018.4628

14. Merlano MC, Merlotti AM, Licitra L, et al.: Activation of immune responses in patients with relapsedmetastatic head and neck cancer (CONFRONT phase I-II trial): multimodality immunotherapy with avelumab, short-course radiotherapy, and cyclophosphamide. Clin Transl Radiat Oncol. 2018, 12:47-52. 10.1016/j.ctro.2018.08.001

15. Bahig H, Aubin F, Stagg J, et al.: Phase I/II trial of Durvalumab plus Tremelimumab and stereotactic body radiotherapy for metastatic head and neck carcinoma. BMC Cancer. 2019, 19:68. 10.1186/s12885-019-5266- 